\title{
Alcohol and Obesity: A Dangerous Association for Fatty Liver Disease
}

\author{
Abdo Mahli Claus Hellerbrand \\ Department of Internal Medicine I, University Hospital Regensburg, Regensburg, Germany
}

\section{Key Words}

Alcoholic liver disease - Nonalcoholic fatty liver disease .

Metabolic syndrome $\cdot$ Obesity $\cdot$ Synergism

\begin{abstract}
Alcoholic liver disease (ALD) and nonalcoholic fatty liver disease (NAFLD) are the most frequent chronic liver disorders, and their advanced forms - alcoholic steatohepatitis and nonalcoholic steatohepatitis - are the most frequent conditions leading to liver cirrhosis and hepatocellular carcinoma worldwide. NAFLD is considered as the hepatic manifestation of the metabolic syndrome. With the pandemic rise of obesity, the incidence of NAFLD is also further increasing, and considering the life style in modern societies, there is a significant overlap of (risk factors causing) NAFLD and (alcohol consumption predisposing for) ALD at least in Western countries. Epidemiological studies propose a causative link between chronic alcohol consumption and progressive liver disease in obese individuals. Furthermore, experimental studies indicate combined pathological effects of alcohol and obesity or fatty acid levels, respectively, on hepatocellular lipid accumulation and injury as well as hepatic inflammation, fibrosis and cancerogenesis. Notably, these combined pathological effects are in part additive but partly even synergistic. And importantly, alcohol does already exhibit synergistic pathological effects with obesity at moderate doses. This indicates significant differences in the dose threshold for hepatotoxic alcohol effects in lean and obese subjects and herewith also has important implications for
\end{abstract}

\section{KARGER}

E-Mail karger@karger.com

www.karger.com/ddi recommendations for 'safe' alcohol consumption. The purpose of this brief review is to update the knowledge on the combined effects of alcohol and obesity on the development and progression of liver disease. Undoubtedly, alcohol and the metabolic syndrome appear as a dangerous mix, and there are important interactive effects of either condition with regard to crucial triggers of liver injury.

(c) 2016 S. Karger AG, Basel

\section{Introduction}

Chronic and excessive alcohol consumption can lead to the development of alcoholic liver disease (ALD), which is a critical health problem in many countries [1]. The spectrum of liver injury ranges from steatosis and steatohepatitis to fibrosis, cirrhosis and hepatocellular carcinoma (HCC). Hepatocellular lipid accumulation is the first pathological step of ALD. Alcohol is predominantly metabolized in hepatocytes, and in the oxidation of ethanol to acetaldehyde, NAD is reduced to NADH, which promotes fatty acid synthesis while counteracting lipid catabolism, thus leading to fat accumulation in hepatocytes $[2,3]$.

More recently, the pathophysiological significance of hepatic lipid accumulation in the absence of significant alcohol consumption is increasingly recognized. Today, nonalcoholic fatty liver disease (NAFLD) is considered the most common cause of liver enzyme elevations in Western countries [4]. In most cases, NAFLD is associ- 


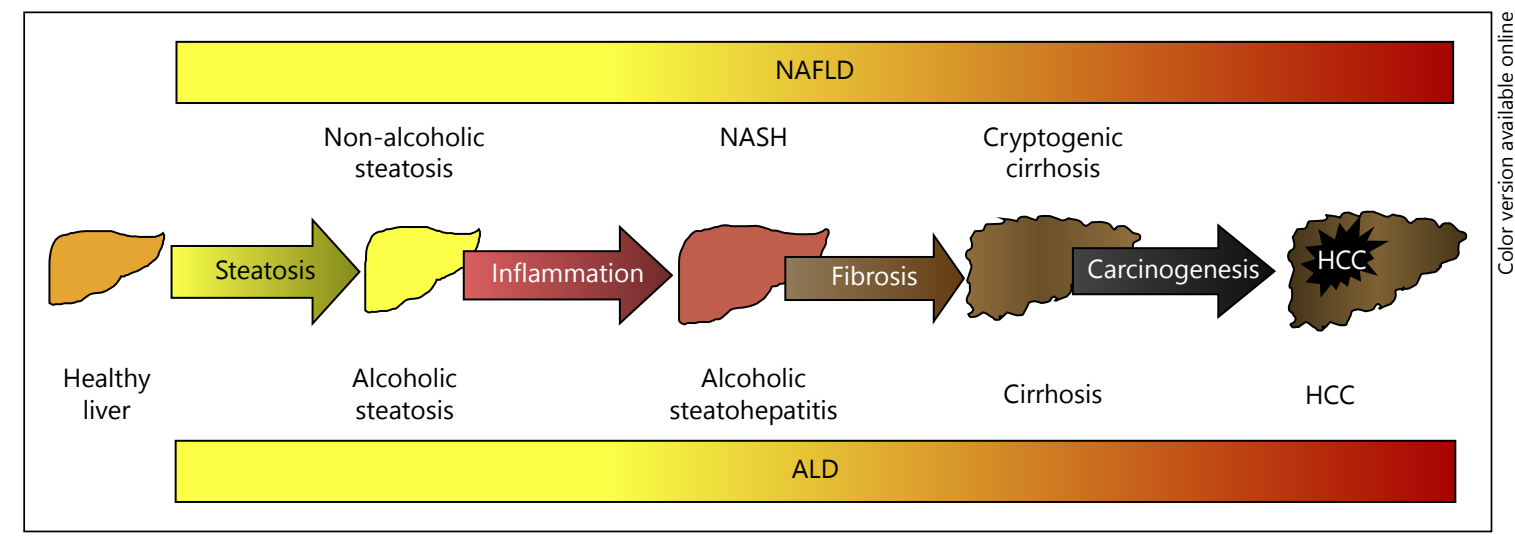

Fig. 1. ALD and NAFLD have very similar pathophysiological steps of disease progression, that is, hepatic steatosis, inflammation and fibrosis. Progressive hepatic fibrosis may ultimately lead to cirrhosis, the major risk factor for HCC.

ated with (components of) the metabolic syndrome, that is, central obesity and insulin resistance with resulting diabetes type 2, dyslipidemia and hypertension. Therefore, NAFLD is regarded as the hepatic manifestation of the metabolic syndrome. Very similar as in ALD, NAFLD encompasses a wide range of pathological conditions from mild hepatic steatosis to steatohepatitis (a syndrome named non-alcoholic steatohepatitis (NASH)) with significant necroinflammation and progressive fibrosis. In its advanced form, NASH is believed to account for a large fraction, if not entirely for what was previously termed 'cryptogenic cirrhosis' and also progresses to HCC in a significant number of cases $[5,6]$. Also, hepatocellular fat accumulation is the first pathological step in NAFLD (fig. 1). The metabolic syndrome is associated with increased levels of circulating free fatty acids (FFA) [7] and the rate of hepatic FFA uptake is not regulated. Therefore, hepatic uptake of FFA is proportional to plasma FFA concentrations and the predominant reason for hepatocellular fat accumulation in NAFLD [8].

\section{Epidemiology of Alcoholic and Non-Alcoholic Fatty Liver Disease}

NAFLD reveals a drastically rising prevalence due to a lifestyle favoring the development of the metabolic syndrome, and today more than half of the adult population in most Western countries has already been found to be overweight. Furthermore, regardless of the precise definition of safe margins for the dose of daily alcohol consumption, certainly, a 2-digit percentage of the population in Western countries chronically consumes alcohol in amounts that predispose for ALD. Therefore, based on statistical numbers, there is a considerable overlap of individuals with both (components of) metabolic syndrome and chronic alcohol consumption. In addition, it has to be considered that in modern societies, people frequently drink alcohol with high caloric foods. Therefore, the overlap of obese and alcohol-drinking individuals is even larger than pure statistics would predict and it may constitute even the vast majority of cases (fig. 2). Certainly, a critical question is the definition of safe margins for the dose alcohol consumption. Dosis facit venenum, and certainly, this is also true for alcohol intake. However, the dose threshold of alcohol for its hepatotoxic effects depends on a variety of factors including ethnicity and gender [9]. Guidelines from the European Association for the Study of the Liver and the American Association for the Study of the Liver recommend $<30 \mathrm{~g}$ of alcohol per day in man and $<20 \mathrm{~g}$ of alcohol per day in women $[9,10]$. Also, the Asian Pacific for the Study of the Liver guideline defines different levels of daily alcohol consumption for men and women ( $<20$ and $10 \mathrm{~g}$, respectively) as 'safe'. So far, body weight and obesity are not considered in guidelines. In fact, existing cutoff levels are used to differentiate ALD from NAFLD in most clinical trials.

\section{Combined Effects of Alcohol and Obesity on the Development and Progression of Liver Disease}

Actually, it appears likely and plausible that the combination of 2 pathological mechanisms causes more harm than either of the 2 factors alone. Thus, it has been shown that pre-existing liver diseases such as hepatitis Cinfection 


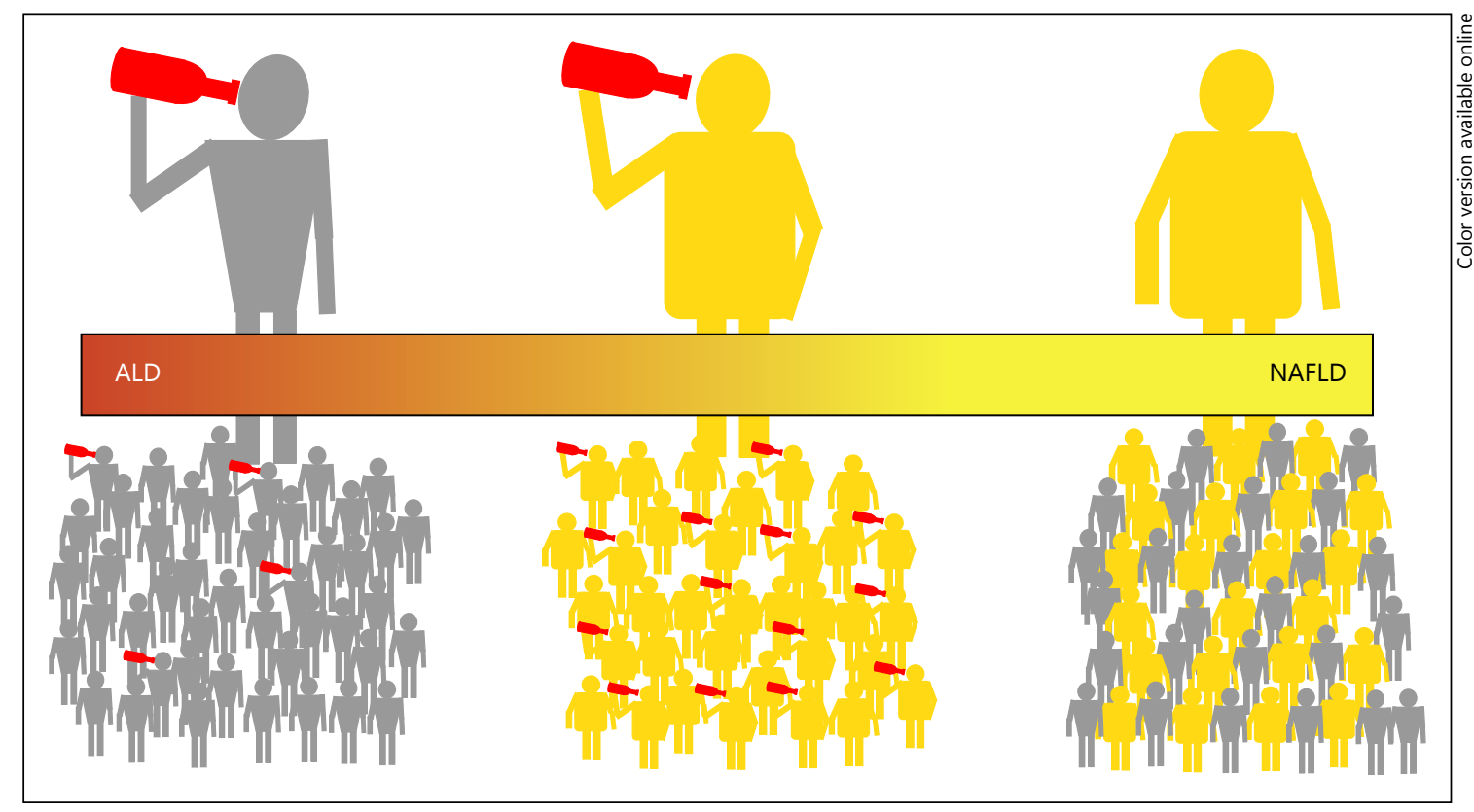

Fig. 2. High frequency and high overlap of chronic alcohol consumption and obesity. A significant percentage of the population in Western countries chronically drinks alcohol in amounts that predispose for ALD (symbolized by a red wine bottle). Furthermore, in many industrialized nations, almost half of the adult pop- ulation are overweight or obese (symbolized by yellow color), and the majority of these individuals have NAFLD. Very frequently, excess alcohol and calorie intake coincide and therefore the frequency of risky alcohol consumption in obese is even higher than in the general population (symbolized by grey color). deteriorate with chronic alcohol ingestion $[11,12]$. Likewise, obesity and diabetes are independent risk factors for liver fibrosis in patients with chronic viral hepatitis infection $[13,14]$. A combined effect on the progression and development of liver injury seems even more probable in the case of alcohol and obesity since ALD and NAFLD reveal astonishing histopathological similarities [15].

Indeed, several epidemiological studies suggest a strong causative link between the consumption of alcohol and progressive liver disease in individuals with high fat intake and/or diabetes. Combined effects appear to affect all pathophysiological steps of disease development and progression, starting with hepatic steatosis. The DIONYSOS study was one of the first epidemiological studies clearly showing combined effect of alcohol consumption and elevated body weight on hepatic steatosis in a large cohort of subjects in Northern Italy [16]. The prevalence of hepatic steatosis determined by ultrasonography was increased in subjects with a daily intake of $>60 \mathrm{~g}$ of alcohol to $46 \%$ and in obese persons to $76 \%$ compared to lean controls that revealed hepatic steatosis only in $16 \%$ of cases. Still, in obese individuals drinking $>60 \mathrm{~g}$ alcohol per day, steatosis was found in even $95 \%$ of individuals [16].
Also, with regards to hepatocellular injury and inflammation, a strong causative link has been found between alcohol consumption and obesity $[17,18]$. Of note, the likelihood of hepatic injury was higher at increasing body weight even when the levels of alcohol consumption were as low $28 \mathrm{~g}$ alcohol per day [17]. Loomba et al. [19] found that the odds for elevated serum transaminase levels increased with higher body mass index (BMI) as well as each alcohol drinking level in a study with 2,364 individuals in the US. OR was approximately 3 in obese people with no or only one drink per day and approximately 2 in lean individuals consuming $40 \mathrm{~g}$ alcohol per day or more. However, OR was nearly 9 in obese individuals drinking $>40 \mathrm{~g}$ alcohol per day, showing a clear synergistic effect of alcohol and obesity on hepatocellular injury.

Alcohol and obesity also appear as a critical association for hepatic fibrosis and development of cirrhosis. In subjects with heavy alcohol consumption, obesity is an independent risk factor for the development of both acute alcohol-induced hepatitis and cirrhosis [20, 21]. Moreover, Ekstedt et al. [22] found an accelerated progression of fibrosis in patients with NAFLD who drank moderate (up to $140 \mathrm{~g} /$ week) amounts of alcohol. Liu et al. [23] analyzed more than 1 million middle aged women in the 
UK. Here, alcohol consumption of $>150$ g/week was found to increase the relative risk for cirrhosis approximately 3 -fold while obesity $(\mathrm{BMI}>30)$ in individuals drinking $<70 \mathrm{~g}$ alcohol/week did not significantly affect the risk for cirrhosis. However, in obese women drinking $>150 \mathrm{~g}$ of alcohol/week, the relative cirrhosis risk increased more than 6 fold.

Lastly, there are strong epidemiological data showing a combined effect of alcohol and obesity on the risk of developing HCC. Ascha et al. [24] found an increasing risk for HCC in patients with underlying NASH who consumed alcohol in moderate amounts. In another study, Loomba et al. [25] analyzed the relative HCC risk in 23,712 Taiwanese residents. In this study, chronic alcohol consumption ( $>4$ times per week for $>1$ year) did not significantly affect HCC risk but obesity increased the risk by approximately 2 fold. Noteworthy, obesity and chronic alcohol consumption together led to a 7 -fold increase of HCC risk in this large study [25] indicating a clear synergistic effect.

\section{Animal Models of Combined Effects of Alcohol and High Fat on Development and Progression of Liver Disease}

Experimental studies have shown that obesity or feeding a high fat diet (HFD), respectively, in combination with alcohol application accelerate hepatic steatosis, inflammation and fibrosis in mice and rats. These experimental models also revealed mechanistic insights on how alcohol and obesity caused synergistic harm.

Carmiel-Haggai et al. [26] showed that short-term binge alcohol exposure increased apoptosis and liver injury in obese rats compared to lean controls. In a study by $\mathrm{Xu}$ et al. [27], moderate obesity induced by intragastric overfeeding of a HFD and alcohol intake caused synergistic steatohepatitis in an alcohol dose-dependent manner. This was associated with increased fibrosis, induction of inducible nitric oxide synthetase and reactive nitrogen species, that is, nitrosative stress. Wang et al. [28] combined a genetic model for NASH, the leptindeficient, insulin-resistant Zucker rat by feeding ethanol containing Lieber-DeCarli diet and identified cytochrome P-4502E1 induction and the generation of carcinogenic DNA lesions as exacerbating pathological forces. Purohit et al. [29] found that combined consumption of alcohol and a HFD significantly increased SREBP-1 and FAS gene expressions in mice. Wang et al. [30] investigated the effect of a rather low intake of ethanol
(16\% of total calories) on the progression of a HFD-induced NASH model in Sprague-Dawley rats. They observed an increased number of inflammatory foci and apoptosis due to the additional intake of ethanol, suggesting that even moderate alcohol intake can augment hepatic inflammation and apoptosis in rodents with underlying NASH.

Also, we established an experimental murine model where we combined feeding a HFD, which results in (modest) weight gain with chronic alcohol administration in the drinking water resembling so-called 'social drinking' [31]. Under these experimental conditions, alcohol and HFD caused additive effects on hepatic steatosis and inflammation, while effects on hepatic fibrosis were clearly synergistic. The pronounced effect on hepatic fibrosis indicates that this phenomenon was not simply a result of timely preceding pathological effects but that alcohol and HFD cause (metabolic) changes that promote fibrogenesis via mechanisms independent of steatosis and inflammation.

\section{An in vitro Model of Combined Effects of Alcohol and Free Fatty on Hepatocytes}

More recently, we have developed an in vitro model to study the combined effects of alcohol and FFA on primary human hepatocytes [32]. To induce intracellular lipid accumulation, cells were incubated with FFA complexed to albumin [33]. Subsequently, steatotic and control hepatocytes were incubated with up to $50 \mathrm{~mm}$ alcohol. This alcohol concentration on its own revealed only minimal effects but significantly enhanced oleate-induced lipogenesis and cellular triglyceride content compared to control cells. Similarly, lipid peroxidation, oxidative stress and pro-inflammatory gene expression were synergistically induced by alcohol and steatosis. Of note, the joint pathological effect of alcohol and cellular triglyceride content were caused by synergistic induction of CYP2E1 activity in our in vitro model. Generally, this cytochrome P450 is known for its detrimental effects in ALD through free radical formation and lipid peroxidation $[34,35]$. Therefore, pharmacological inhibition of CYP2E1 has emerged as strategy for treatment of alcoholinduced liver injury $[34,36]$. Also, several studies identified the induction of cytochrome P4502E1 as a critical pathological factor in NAFLD. This cytochrome metabolized both fatty acids and ethanol. When these substances are chronically present in large amounts, they induce the activity of this enzyme which is associated with the release 
of free radicals. This reaction can cause lipid peroxidation and liver injury, and in vitro data indicate that these mechanisms are even synergistically enhanced in the presence of both alcohol and excessive FFA.

Notably, alcohol and cellular steatosis also induced autophagy in a synergistic manner in hepatocytes in vitro, and this was also mediated via CYP2E1 [32]. Further induction of autophagy ameliorated the joint effects of alcohol and oleic acid on hepatocellular lipid accumulation and inflammatory gene expression while inhibition of autophagy further enhanced the dual pathological effects.

Autophagy is a highly conserved intracellular catabolic pathway for the degradation of long-lived proteins and cytoplasmic organelles. The effects of ethanol on autophagy are complex and only partly understood but it is becoming clear that autophagy serves a protective function against alcohol-induced liver injury [37, 38]. Similarly, there is increasing evidence that autophagy also inhibits NAFLD progression [39-41].

Certainly, the clinical relevancy of our in vitro findings in hepatocytes has to be verified. Still, the CYP2E1mediated joint effects of alcohol- and oleate-induced steatosis on autophagy indicate that alcohol induces not only pathological but also protective mechanisms in steatotic hepatocytes via CYP2E1. Therefore, the manipulation of CYP2E1 may be a double-edged sword and warrant the exercise of caution in the pharmacological use of CYP2E1 inhibitors for the treatment of ALD in obese individuals. Furthermore, one may speculate whether individual factors tipping the balance on the one or the other side of detrimental or beneficial joint effects of alcohol and FFA account at least in part for the high variation in the clinical course of ALD. Only slight differences in the ratio of individual beneficial and detrimental factors may decide whether moderate alcohol consumption is protective or is causing harm in (non-alcoholic) fatty livers. Actually, there are some studies suggesting that moderate alcohol consumption might even be protective for patients with NAFLD $[42,43]$. Light to moderate alcohol consumption can lead to an improvement in peripheral insulin resistance, and herewith, may also have a protective effect against the development of diabetes and hepatic steatosis [44-47]. However, these studies have to be reflected on with great caution. The border between safe or eventually even beneficial alcohol consumption on the one side and dangerous and deleterious levels on the other side is extremely narrow and depends also on several confounding variables which are only incompletely understood. Furthermore, one needs to be cautious because even if a certain level of drinking might protect against fatty liver disease, it may be harmful for other organs and tissues and may also increase the risk of certain cancers, such as breast or colorectal cancer [48, 49].

\section{Extrahepatic Mechanisms Affecting Disease Progression in ALD and NAFLD}

It has to be noted that both ALD and NAFLD occur in the context of a systemic disease, that is, (components of) the metabolic syndrome and chronic alcoholism. Therefore, in addition to the liver, several other organs and biological functions can be affected. These pathological alterations can directly or indirectly promote inflammation and fibrosis in fatty liver [50]. For instance, alcohol and obesity/diabetes lead to quantitative and qualitative changes of the microbiome and an impaired intestinal barrier, and these mechanisms are known to promote hepatic inflammation, fibrosis and cancerogenesis [51, 52]. One further example is (visceral) adipose tissue, in which secretion of adipokines is altered by both obesity and alcohol. Adiponectin, which is known to be hepatoprotective and anti-fibrotic, is reduced in individuals with obesity or chronic alcohol consumption [53-55]. In contrast, both chronic alcohol intake and obesity induce the expression of the profibrogenic adipokine leptin in adipose tissue $[56,57]$. There are several more extrahepatic systemic processes and biological functions affected by both alcohol and the metabolic syndrome, making the relationship between ALD and NAFLD extremely complex. Accordingly, the mechanisms responsible for accelerated disease progression if both conditions coincide are most likely related to multiple factors.

\section{Confounding Dietary Factors Affecting Disease Progression in ALD and NAFLD}

Experimental and epidemiological studies clearly indicate that in addition to the quantity, the type of dietary fat also critically affects the pathogenesis of ALD $[58,59]$ and NAFLD [60, 61]. For instance, in vitro studies clearly show that saturated FFA cause more apoptosis in hepatocytes than unsaturated FFA $[62,63]$. Besides lipids, quantity and quality of carbohydrates consumption have been shown to affect hepatic steatosis, inflammation and fibrosis in experimental models $[64,65]$. These findings are of particular relevancy in ALD where malnutrition is a frequently observed problem [66].
36

Dig Dis 2016;34(suppl 1):32-39

DOI: $10.1159 / 000447279$
Mahli/Hellerbrand 
Moreover, it becomes more and more evident that not only the amount of consumed alcohol but also the subcategory of alcoholic beverage affects the development and progression liver disease. A recent study found that the ratio of the budget for healthy to that for unhealthy foods were highest for wine drinkers and lowest for beer drinkers [67]. In this study, healthy foods included for instance coffee, and it has been shown by others that coffee consumption has hepatoprotective effects $[68,69]$. On the other side, unhealthy foods were considered for instance as soft drinks and saturated fatty acids, that is, dietary components well known to accelerate progression of liver disease $[63,70]$. Some studies have also suggested that a high percentage of red wine in the amount of alcohol consumed lowers the relative risk to develop (alcoholic) liver cirrhosis [71]. One explanation for this phenomenon may be resveratrol, a polyphenol in grapes, which is also found in red wines [72]. Several studies have demonstrated the protective effects of resveratrol in experimental models of liver injury in animals [73-75]. More recent studies show that beer also contains substances that protect from liver damage [76]. Furthermore, experimental studies revealed that hop ingredients such as xanthohumol or iso-alpha acids inhibit hepatic steatosis, inflammation, fibrosis and hepatocarcinogenesis [77]. Moreover, these hop ingredients have been shown to beneficially affect the metabolic syndrome [77] and it has been shown already in patients with prediabetes that doses of iso-alpha acids as low as $1.5 \mathrm{mg} / \mathrm{kg}$ daily improved insulin resistance and reduced body weight [78]. This dose corresponds to a beer consumption of approximately 1-6 liter depending on the type of beer [79]. This means that with alcohol consumption of approximately $40 \mathrm{~g} /$ day in the form of beers with high content of isoalpha acids, some beneficial effects on insulin resistance and body weight could be achieved. Together, the available data show that not only the amount of alcohol and calorie intake but also the type of food and alcoholic beverages affect ALD and NAFLD, which further contributes to the complexity of the interaction between alcohol and obesity in (chronic) liver disease.

\section{Conclusion}

Alcoholic and non-alcoholic liver fatty liver disease are the most frequent liver diseases worldwide, and one needs to consider that both conditions overlap in a significant number of patients. There is unequivocal epidemiological and experimental evidence that alcohol and (components of) the metabolic syndrome exhibit combined effects on the development and progression of liver injury, which are in part additive and partly even synergistic. Consequently, these combined effects have a major impact on the dose threshold for hepatotoxic alcohol effects, that is, safety levels for 'social drinking' and guideline recommendations for 'safe' alcohol consumption. Today, practically all guideline recommendations define different threshold levels for hepatotoxic alcohol in man and woman. Available data strongly suggest that in addition to gender, the presence of overweight and obesity should be considered for defining safe alcohol levels. Obese individuals should adhere to lower amounts of regular alcohol consumption than lean subjects, and those who drink within safe margins should take care to not become overweight.

\section{Disclosure Statement}

Both authors have no conflicts of interest to declare.

\section{References}

1 Ramaiah S, Rivera C, Arteel G: Early-phase alcoholic liver disease: an update on animal models, pathology, and pathogenesis. Int J Toxicol 2004;23:217-231.

2 Lieber CS, Schmid R: The effect of ethanol on fatty acid metabolism; stimulation of hepatic fatty acid synthesis in vitro. J Clin Invest 1961; 40:394-399.

3 Galli A, Price D, Crabb D: High-level expression of rat class I alcohol dehydrogenase is sufficient for ethanol-induced fat accumulation in transduced HeLa cells. Hepatology 1999;29:1164-1170.

4 Clark JM, Brancati FL, Diehl AM: The prevalence and etiology of elevated aminotransfer- ase levels in the United States. Am J Gastroenterol 2003;98:960-967.

5 Rozman D: From nonalcoholic fatty liver disease to hepatocellular carcinoma: a systems understanding. Dig Dis Sci 2014;59:238-241.

6 Rinella ME: Nonalcoholic fatty liver disease: a systematic review. JAMA 2015;313:22632273.

7 Kusminski CM, Shetty S, Orci L, Unger RH, Scherer PE: Diabetes and apoptosis: lipotoxicity. Apoptosis 2009;14:1484-1495.

8 Teli MR, James OF, Burt AD, Bennett MK, Day CP: The natural history of nonalcoholic fatty liver: a follow-up study. Hepatology 1995;22:1714-1719.
9 European Association for the Study of Liver: EASL clinical practical guidelines: management of alcoholic liver disease. J Hepatol 2012;57:399-420.

10 O'Shea RS, Dasarathy S, McCullough AJ; Practice Guideline Committee of the American Association for the Study of Liver Diseases; Practice Parameters Committee of the American College of Gastroenterology: Alcoholic liver disease. Hepatology 2010;51: 307-328.

11 Mueller S, Millonig G, Seitz HK: Alcoholic liver disease and hepatitis C: a frequently underestimated combination. World J Gastroenterol 2009; 15:3462-3471. 
12 Lederer SL, Walters KA, Proll S, Paeper B, Robinzon S, Boix L, et al: Distinct cellular responses differentiating alcohol- and hepatitis C virus-induced liver cirrhosis. Virol J 2006; 3:98.

13 Hourigan LF, Macdonald GA, Purdie D, Whitehall VH, Shorthouse C, Clouston A, et al: Fibrosis in chronic hepatitis $\mathrm{C}$ correlates significantly with body mass index and steatosis. Hepatology 1999;29:1215-1219.

14 Cimino L, Oriani G, D’Arienzo A, Manguso F, Loguercio C, Ascione A, et al: Interactions between metabolic disorders (diabetes, gallstones, and dyslipidaemia) and the progression of chronic hepatitis $\mathrm{C}$ virus infection to cirrhosis and hepatocellular carcinoma. A cross-sectional multicentre survey. Dig Liver Dis 2001;33:240-246.

15 Falck-Ytter Y, Younossi ZM, Marchesini G, McCullough AJ: Clinical features and natural history of nonalcoholic steatosis syndromes. Semin Liver Dis 2001;21:17-26.

16 Bellentani S, Saccoccio G, Masutti F, Croce LS, Brandi G, Sasso F, et al: Prevalence of and risk factors for hepatic steatosis in Northern Italy. Ann Intern Med 2000;132:112-117.

17 Ruhl CE, Everhart JE: Joint effects of body weight and alcohol on elevated serum alanine aminotransferase in the United States population. Clin Gastroenterol Hepatol 2005;3: 1260-1268.

18 Alatalo PI, Koivisto HM, Hietala JP, Puukka KS, Bloigu R, Niemela OJ: Effect of moderate alcohol consumption on liver enzymes increases with increasing body mass index. Am J Clin Nutr 2008;88:1097-1103.

19 Loomba R, Bettencourt R, Barrett-Connor E: Synergistic association between alcohol intake and body mass index with serum alanine and aspartate aminotransferase levels in older adults: the Rancho Bernardo study. Aliment Pharmacol Ther 2009;30:1137-1149.

20 Naveau S, Giraud V, Borotto E, Aubert A, Capron F, Chaput JC: Excess weight risk factor for alcoholic liver disease. Hepatology 1997;25:108-111.

21 Raynard B, Balian A, Fallik D, Capron F, Bedossa P, Chaput JC, et al: Risk factors of fibrosis in alcohol-induced liver disease. Hepatology 2002;35:635-638.

22 Ekstedt M, Franzen LE, Holmqvist M, Bendtsen P, Mathiesen UL, Bodemar G, et al: Alcohol consumption is associated with progression of hepatic fibrosis in non-alcoholic fatty liver disease. Scand J Gastroenterol 2009;44:366-374.

23 Liu B, Balkwill A, Reeves G, Beral V; Million Women Study Collaborators: Body mass index and risk of liver cirrhosis in middle aged UK women: prospective study. BMJ 2010; 340:c912.

24 Ascha MS, Hanouneh IA, Lopez R, Tamimi TA, Feldstein AF, Zein NN: The incidence and risk factors of hepatocellular carcinoma in patients with nonalcoholic steatohepatitis. Hepatology 2010;51:1972-1978.

25 Loomba R, Yang HI, Su J, Brenner D, BarrettConnor E, Iloeje U, et al: Synergism between obesity and alcohol in increasing the risk of hepatocellular carcinoma: a prospective cohort study. Am J Epidemiol 2013;177:333342.

26 Carmiel-Haggai M, Cederbaum AI, Nieto N: Binge ethanol exposure increases liver injury in obese rats. Gastroenterology 2003; 125 : $1818-1833$.

27 Xu J, Lai KK, Verlinsky A, Lugea A, French SW, Cooper MP, et al: Synergistic steatohepatitis by moderate obesity and alcohol in mice despite increased adiponectin and p-AMPK. J Hepatol 2011;55:673-682.

28 Wang Y, Millonig G, Nair J, Patsenker E, Stickel F, Mueller S, et al: Ethanol-induced cytochrome P4502E1 causes carcinogenic etheno-DNA lesions in alcoholic liver disease. Hepatology 2009;50:453-461.

29 Purohit V, Gao B, Song BJ: Molecular mechanisms of alcoholic fatty liver. Alcohol Clin Exp Res 2009;33:191-205.

30 Wang Y, Seitz HK, Wang XD: Moderate alcohol consumption aggravates high-fat diet induced steatohepatitis in rats. Alcohol Clin Exp Res 2010;34:567-573.

31 Gabele E, Dostert K, Dorn C, Patsenker E, Stickel F, Hellerbrand C: A new model of interactive effects of alcohol and high-fat diet on hepatic fibrosis. Alcohol Clin Exp Res 2011; 35:1361-1367.

32 Mahli A, Thasler WE, Patsenker E, Muller S, Stickel F, Muller M, et al: Identification of cytochrome CYP2E1 as critical mediator of synergistic effects of alcohol and cellular lipid accumulation in hepatocytes in vitro. Oncotarget 2015;6:41464-41478.

33 Wobser H, Dorn C, Weiss TS, Amann T, Bollheimer C, Buttner R, et al: Lipid accumulation in hepatocytes induces fibrogenic activation of hepatic stellate cells. Cell Res 2009;19:9961005.

34 Gebhardt AC, Lucas D, Menez JF, Seitz HK: Chlormethiazole inhibition of cytochrome P450 2E1 as assessed by chlorzoxazone hydroxylation in humans. Hepatology 1997;26: 957-961.

$35 \mathrm{Wu} \mathrm{D}$, Wang X, Zhou R, Cederbaum A: CYP2E1 enhances ethanol-induced lipid accumulation but impairs autophagy in HepG2 E47 cells. Biochem Biophys Res Commun 2010;402:116-122.

36 Swaminathan K, Clemens DL, Dey A: Inhibition of CYP2E1 leads to decreased malondialdehyde-acetaldehyde adduct formation in VL-17A cells under chronic alcohol exposure. Life Sci 2013;92:325-336.

37 Ding WX, Li M, Chen X, Ni HM, Lin CW, Gao W, et al: Autophagy reduces acute ethanol-induced hepatotoxicity and steatosis in mice. Gastroenterology 2010;139:1740-1752.

38 Lin CW, Zhang H, Li M, Xiong X, Chen X, Chen X, et al: Pharmacological promotion of autophagy alleviates steatosis and injury in alcoholic and non-alcoholic fatty liver conditions in mice. J Hepatol 2013;58:993-999.

39 Singh R, Kaushik S, Wang Y, Xiang Y, Novak I, Komatsu M, et al: Autophagy regu- lates lipid metabolism. Nature 2009;458: 1131-1135.

40 Czaja MJ: Autophagy in health and disease. 2. Regulation of lipid metabolism and storage by autophagy: pathophysiological implications. Am J Physiol Cell Physiol 2010;298:C973C978.

41 Gonzalez-Rodriguez A, Mayoral R, Agra N, Valdecantos MP, Pardo V, Miquilena-Colina $\mathrm{ME}$, et al: Impaired autophagic flux is associated with increased endoplasmic reticulum stress during the development of NAFLD. Cell Death Dis 2014;5:e1179.

42 Sookoian S, Castano GO, Pirola CJ: Modest alcohol consumption decreases the risk of nonalcoholic fatty liver disease: a meta-analysis of 43175 individuals. Gut 2014;63:530-532.

43 Dunn W, Sanyal AJ, Brunt EM, Unalp-Arida A, Donohue M, McCullough AJ, et al: Modest alcohol consumption is associated with decreased prevalence of steatohepatitis in patients with non-alcoholic fatty liver disease (NAFLD). J Hepatol 2012;57:384-391.

44 Wannamethee SG, Camargo CA Jr, Manson JE, Willett WC, Rimm EB: Alcohol drinking patterns and risk of type 2 diabetes mellitus among younger women. Arch Intern Med 2003;163:1329-1336.

45 Moriya A, Iwasaki Y, Ohguchi S, Kayashima E, Mitsumune T, Taniguchi $\mathrm{H}$, et al: Alcohol consumption appears to protect against nonalcoholic fatty liver disease. Aliment Pharmacol Ther 2011;33:378-388.

46 Gunji T, Matsuhashi N, Sato H, Fujibayashi $\mathrm{K}$, Okumura M, Sasabe N, et al: Light and moderate alcohol consumption significantly reduces the prevalence of fatty liver in the Japanese male population. Am J Gastroenterol 2009; 104:2189-2195.

47 Dunn W, Xu R, Schwimmer JB: Modest wine drinking and decreased prevalence of suspected nonalcoholic fatty liver disease. Hepatology 2008;47:1947-1954.

48 Seitz HK: Alcohol and breast cancer. Breast 2012;21:426-427.

49 Homann N, Konig IR, Marks M, Benesova M, Stickel F, Millonig G, et al: Alcohol and colorectal cancer: the role of alcohol dehydrogenase 1C polymorphism. Alcohol Clin Exp Res 2009;33:551-556.

50 Hellerbrand C: Pathophysiological similarities and synergisms in alcoholic and non-alcoholic steatohepatitis. Dig Dis 2010;28:783791

51 Yan AW, Schnabl B: Bacterial translocation and changes in the intestinal microbiome associated with alcoholic liver disease. World J Hepatol 2012;4:110-118.

52 Szabo G: Gut-liver axis in alcoholic liver disease. Gastroenterology 2015;148:30-36.

53 Finelli C, Tarantino G: What is the role of adiponectin in obesity related non-alcoholic fatty liver disease? World J Gastroenterol 2013; 19:802-812.

54 Rogers CQ, Ajmo JM, You M: Adiponectin and alcoholic fatty liver disease. IUBMB Life 2008;60:790-797. 
55 Wang Y, Zhou M, Lam KS, Xu A: Protective roles of adiponectin in obesity-related fatty liver diseases: mechanisms and therapeutic implications. Arq Bras Endocrinol Metabol 2009;53:201-212.

56 Rajkovic N, Zamaklar M, Lalic K, Jotic A, Lukic L, Milicic T, et al: Relationship between obesity, adipocytokines and inflammatory markers in type 2 diabetes: relevance for cardiovascular risk prevention. Int J Environ Res Public Health 2014;11:4049-4065.

57 Saxena NK, Titus MA, Ding X, Floyd J, Srinivasan S, Sitaraman SV, et al: Leptin as a novel profibrogenic cytokine in hepatic stellate cells: mitogenesis and inhibition of apoptosis mediated by extracellular regulated kinase (Erk) and Akt phosphorylation. FASEB J 2004;18:1612-1614.

58 Nanji AA, French SW: Relationship between pork consumption and cirrhosis. Lancet 1985; 1:681-683.

59 Nanji AA, Mendenhall CL, French SW: Beef fat prevents alcoholic liver disease in the rat. Alcohol Clin Exp Res 1989;13:15-19.

60 Buettner R, Parhofer KG, Woenckhaus M, Wrede CE, Kunz-Schughart LA, Scholmerich $\mathrm{J}$, et al: Defining high-fat-diet rat models: metabolic and molecular effects of different fat types. J Mol Endocrinol 2006;36:485-501.

61 Tipoe GL, Ho CT, Liong EC, Leung TM, Lau TY, Fung ML, et al: Voluntary oral feeding of rats not requiring a very high fat diet is a clinically relevant animal model of non-alcoholic fatty liver disease (NAFLD). Histol Histopathol 2009;24:1161-1169.

62 Gentile CL, Pagliassotti MJ: The role of fatty acids in the development and progression of nonalcoholic fatty liver disease. J Nutr Biochem 2008;19:567-576.
63 Wang D, Wei Y, Pagliassotti MJ: Saturated fatty acids promote endoplasmic reticulum stress and liver injury in rats with hepatic steatosis. Endocrinology 2006;147:943-951.

64 Bergheim I, Weber S, Vos M, Kramer S, Volynets V, Kaserouni S, et al: Antibiotics protect against fructose-induced hepatic lipid accumulation in mice: role of endotoxin. J Hepatol 2008;48:983-992.

65 Feldstein AE, Canbay A, Guicciardi ME, Hi guchi $\mathrm{H}$, Bronk SF, Gores GJ: Diet associated hepatic steatosis sensitizes to Fas mediated liver injury in mice. J Hepatol 2003;39:978983.

66 Bergheim I, Parlesak A, Dierks C, Bode JC, Bode C: Nutritional deficiencies in German middle-class male alcohol consumers: relation to dietary intake and severity of liver disease. Eur J Clin Nutr 2003;57:431-438.

67 Hansel B, Roussel R, Diguet V, Deplaude A, Chapman MJ, Bruckert E: Relationships between consumption of alcoholic beverages and healthy foods: the French supermarket cohort of 196,000 subjects. Eur J Prev Cardiol 2015;22:215-222

68 Chen S, Teoh NC, Chitturi S, Farrell GC: Coffee and non-alcoholic fatty liver disease: brewing evidence for hepatoprotection? J Gastroenterol Hepatol 2014;29:435-441.

69 Liu F, Wang X, Wu G, Chen L, Hu P, Ren H, et al: Coffee consumption decreases risks for hepatic fibrosis and cirrhosis: a meta-analysis. PLoS One 2015; 10:e0142457.

70 Assy N, Nasser G, Kamayse I, Nseir W, Beniashvili Z, Djibre A, et al: Soft drink consumption linked with fatty liver in the absence of traditional risk factors. Can J Gastroenterol 2008:22:811-816

71 Becker U, Gronbaek M, Johansen D, Sorensen TI: Lower risk for alcohol-induced cirrhosis in wine drinkers. Hepatology 2002;35:868875
72 Baur JA, Sinclair DA: Therapeutic potential of resveratrol: the in vivo evidence. Nat Rev Drug Discov 2006;5:493-506.

73 Kasdallah-Grissa A, Mornagui B, Aouani E Hammami M, Gharbi N, Kamoun A, et al: Protective effect of resveratrol on ethanol-induced lipid peroxidation in rats. Alcohol Alcohol 2006;41:236-239.

74 Bujanda L, Garcia-Barcina M, Gutierrez-de Juan V, Bidaurrazaga J, de Luco MF, Gutierrez-Stampa M, et al: Effect of resveratrol on alcohol-induced mortality and liver lesions in mice. BMC Gastroenterol 2006;6:35.

75 Ajmo JM, Liang X, Rogers CQ, Pennock B, You M: Resveratrol alleviates alcoholic fatty liver in mice. Am J Physiol Gastrointest Liver Physiol 2008;295:G833-G842.

76 Kanuri G, Wagnerberger S, Landmann M, Prigl E, Hellerbrand C, Bischoff SC, et al: Effect of acute beer ingestion on the liver: studies in female mice. Eur J Nutr 2015;54:465474

77 Weiskirchen R, Mahli A, Weiskirchen S, Hellerbrand C: The hop constituent xanthohumol exhibits hepatoprotective effects and inhibits the activation of hepatic stellate cells at different levels. Front Physiol 2015;6:140.

78 Obara K, Mizutani M, Hitomi Y, Yajima H, Kondo K: Isohumulones, the bitter component of beer, improve hyperglycemia and decrease body fat in Japanese subjects with prediabetes. Clin Nutr 2009;28:278-284.

79 Heyerick A, Zhao Y, Sandra P, Huvaere K, Roelens F, De Keukeleire D: Photolysis of hop-derived trans-iso-alpha-acids and transtetrahydroiso-alpha-acids: product identification in relation to the lightstruck flavour of beer. Photochem Photobiol Sci 2003;2:306314 